\title{
Schedule-induced vs. home cage ethanol consumption: Influence of food deprivation
}

\author{
MINDA R. LYNCH, JOSEPH H. PORTER, and JOHN A. ROSECRANS \\ Departments of Psychology and Pharmacology \\ Virginia Commonwealth University, Richmond, Virginia 23284
}

\begin{abstract}
Four rats at $80 \%$ of their free-feeding body weights received six daily 1-h sessions of scheduleinduced polydipsia training with $9.5 \%$ (v/v) ethanol solution on a fixed-time 2-min food schedule for a total of 20 days. Then choice testing with ethanol vs. water was conducted for 10 days during the polydipsia sessions. Next, the four rats were returned to home cages, where choice testing continued for 7 days under ad-lib feeding conditions and then 10 days under reinstated food-deprivation conditions. While ethanol consumption was high during both polydipsia and home cage choice testing at $80 \%$ body weight, it decreased to nearly zero when the rats were given ad-lib access to food in the home cage choice testing condition. These data support a "caloric hypothesis" interpretation of ethanol ingestion in rats.
\end{abstract}

Lester and Freed (1973) have argued that an adequate animal model of alcoholism must include excessive oral consumption of ethanol when competing fluids are available and also when the animals are not food deprived. Although several techniques of alcohol administration have been successful in achieving tolerance and physical dependence (see Mello, 1973), only scheduleinduced polydipsia (SIP) (Falk, 1961) produces the excessive consumption required by these criteria. Falk, Samson, and Winger (1976) provided rats with $(5 \% \mathrm{v} / \mathrm{v})$ ethanol during 3 months of polydipsia testing and reported a consumption rate of $13.1 \mathrm{~g} / \mathrm{kg} / \mathrm{day}$, which resulted in blood alcohol levels sufficient for the development of physical dependence.

Although Falk and Samson (1976) report high preferences for ethanol after only 12 days of dosing with a SIP regimen, other studies suggest that ethanol ingestion in this paradigm is not directed toward the central reinforcing properties of the drug. Heintzelman, Best, and Senter (1976) replicated Falk, Samson, and Winger's (1972) procedure, yet failed to show a preference for ethanol following 3 months of chronic administration. Similarly, Myers, Stoltman, and Martin (1972) had previously demonstrated withdrawal signs in rhesus monkeys after $6-\mathrm{mg} / \mathrm{kg} /$ day dosing for 18 days, and yet they also failed to show a preference for ethanol. Further complicating this issue of an induced preference for ethanol, Keehn and Coulson (1975) reported a preference for concentrations of ethanol up to $9 \%(\mathrm{v} / \mathrm{v})$ in animals deprived to $80 \%$ ad-lib body weight and merely allowed access to ethanol in the home cage. This

This research was supported in part by a Virginia Commonwealth University faculty grant-in-aid to J. H. Porter. The authors would like to thank Mark G. Burnet for his assistance in collecting data for this study. Send reprint requests to J. H. Porter, Psychology Department, Virginia Commonwealth University, 810 West Franklin Street, Richmond, Virginia 23284. and similar findings (Freed, 1972, 1974; Freed \& Lester, 1970) have prompted the formation of a "caloric" hypothesis (Freed, 1972) that proposes that ethanol ingestion by laboratory animals is directed to the nutritive properties of the substance, rather than to its intoxicating effects.

In the present study, rats were trained on Falk et al.'s (1972) original SIP paradigm for 20 days and tested for ethanol preference in a choice test. Then, choice testing was continued in home cages under both ad-lib and food-deprivation conditions in order to assess the importance of schedule induction and food deprivation in ethanol consumption preference.

\section{METHOD}

Animals

One female albino and three male adult albino rats (SpragueDawley strain) were used. They were 120 days old at the initiation of the experiment and weighed from 314 to $457 \mathrm{~g}$.

\section{Experimental Environment}

SIP training and choice testing were conducted in Scientific Prototype (Model 100-A) operant chambers. The lever was removed from each chamber, and the chambers were housed in sound-attenuating cubicles. Standard formula pellets $(45-\mathrm{mg}$ Noyes) were delivered during polydipsia sessions. A stainless steel drinking spout entered on each side of the food magazine, and house lighting was provided in the chamber during all test sessions.

Animals were individually housed in standard stainless steel rat cages during home cage testing. Ad-lib access to water and ethanol solutions was provided from two bottles mounted on the front of the cage, and food was placed on the floor of the cage. Animals were maintained on a 12-h light/dark cycle.

\section{Procedure}

At 90 days of age, these animals received 201 -h sessions of SIP training followed by 20 sessions with $9.5 \%(\mathrm{v} / \mathrm{v})$ ethanol only. They were then subjected to 18 sessions of choice testing with water and ethanol, the last 8 of which were under ad-lib food conditions. 
Following this pilot testing, they were maintained on ad-lib food (Purina Lab Chow) and water in the home cage for 1 month. They were then reduced to $80 \%$ of their new free-feeding body weights by limiting food rations for 9 days and were maintained at this reduced body weight throughout the experiment, except where noted.

Schedule-induced condition. Animals received 20 days of SIP training, with $9.5 \%(\mathrm{v} / \mathrm{v})$ ethanol as the sole fluid in the experimental chamber (SIP-E). Training consisted of noncontingent pellet delivery on a fixed-time (FT) 2-min schedule for six daily 1-h sessions as described by Falk et al. (1972). Total milliliter intake was recorded, body weights were obtained, and food supplements were provided each day between 1200 and $1400 \mathrm{~h}$. The position of the ethanol solution was alternated each day to control for position preference.

Following the training regimen, two-bottle choice testing was conducted in the experimental chamber by providing water vs. 9.5\% ethanol for the entire 24 -h period (SIP-CT). This testing was continued for 10 days with the six daily SIP sessions.

Home cage conditions. At the end of choice testing under the SIP procedure, animals were returned to home cages and provided with ad-lib food. Choice testing was continued in this environment for 7 days (HC-AL). On Day 8, animals were again food deprived and placed on limited food rations for another 10 days. During this time, choice testing continued in the home cages (HC-FD), and all animals returned to their $80 \%$ ad-lib body weights by the end of the 10-day period.

\section{RESULTS AND DISCUSSION}

As seen in Figure 1, ethanol consumption was high during both polydipsia (SIP-CT) and home cage (HC-FD) conditions when rats were maintained at $80 \%$ body weight. Reinstatement of ad-lib access to food in the home cage (HC-AL) resulted in a drop in mean ethanol intake to nearly zero. Treatment by subject analyses of variance for the milliliters of ethanol intake and the percent ethanol intake over the last 3 days of each testing condition were both significant $[F(3,9)=24.7$, $\mathrm{p}<.001$, and $\mathrm{F}(2,6)=14.9, \mathrm{p}<.005$, respectively] Post hoc comparisons with a Tukey HSD test revealed that absolute ethanol intake during ad-lib feeding conditions (HC-AL) was significantly less $(p<.01)$ than during all food-deprivation conditions (SIP-E, SIP-CT, and HC-FD). The percent ethanol intake for HC-AL testing was also significantly less $(p<.01)$ than during SIP-CT or HC-FD conditions.

During the SIP-CT condition, Rats A-2 and A-4 showed a definite preference for the ethanol solution over water (91.4\% and $93.3 \%$, respectively). For A-1 and A-3, ethanol consumption represented only $26.8 \%$ and $35.0 \%$ of the total fluid consumption; however, it should be noted that neither rat showed any decline in absolute ethanol intake in the SIP-CT condition $(27.2 \mathrm{ml}$ and $46.0 \mathrm{ml}$, respectively) as compared with the SIP-E condition $(27.2 \mathrm{ml}$ and $34.1 \mathrm{ml})$. All four rats showed a preference for ethanol over water during the HC-FD condition, with percentages ranging from $76.7 \%$ to $81.7 \%$.

Expressed in mean grams per kilogram per day dose, ethanol intake over the last 3 days of each condition was 9.0 for SIP-E and rose slightly to 10.7 during SIP-CT. These doses are similar to those previously reported (10.3 to 15.1) in ethanol SIP studies (Falk

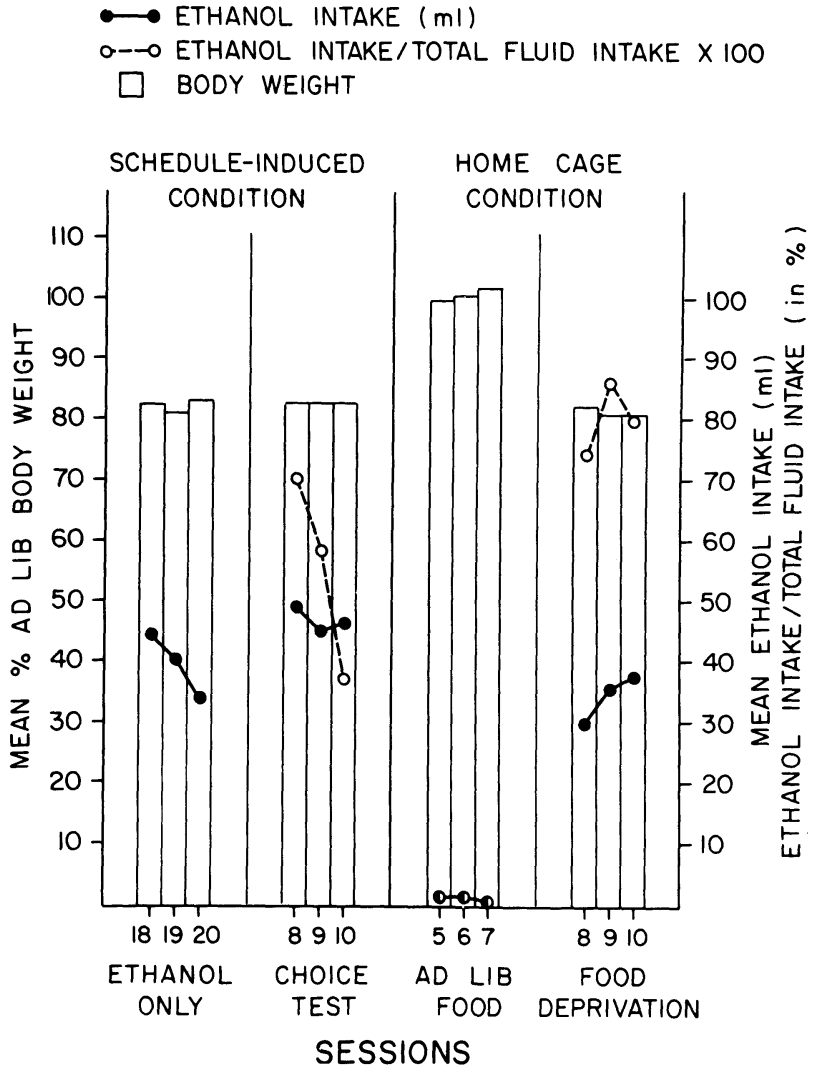

Figure 1. Mean ethanol intake (in milliliters), mean percent ethanol intake [ethanol intake/( $\mathrm{ml}$ ethanol $+\mathrm{ml}$ water $)$ ] $\times 100$, and mean percent of ad-lib body weights are shown for the last 3 days of each testing condition.

\& Samson, 1976; Falk et al., 1976; Heintzelman et al., 1976; Roehrs \& Samson, 1980; Samson \& Falk, 1974a, 1974b; Tang \& Falk, 1977). Under HC-AL conditions, animals only took $.3 \mathrm{mg} / \mathrm{kg} /$ day ethanol, but this dose rose to a mean of 7.8 during food-deprivation conditions in HC-FD testing. As with the other two measures of ethanol intake, the analysis of variance for these data was significant $[F(3,9)=24.6, p<.001]$, and pairwise comparisons indicated significant differences $(p<.01)$ between HC-AL conditions and the other three.

These data support earlier reports of decreased ethanol intake with the return of free-feeding body weights in the home cage situation (Westerfeld \& Lawrow, 1953) and in operant chambers when animals were barpressing for ethanol (Meisch \& Thompson, 1973, 1974). Thus, they indicate the importance of food deprivation in ethanol consumption and suggest additional consideration of the "caloric hypothesis" (Freed, 1972) as a plausible explanation for such self-administration in laboratory rats. They also parallel the results of Heintzelman et al. (1976), who reported that rats provided with unlimited food rations after termination of polydipsia training failed to demonstrate a preference for ethanol. Even Samson and Falk (1974a) have reported a drop in mean daily ethanol dose during polydipsia sessions when body weights are allowed to increase. 
Additional support for the role of deprivation on ethanol consumption in the present study is provided by the observation that as body weights gradually dropped to $80 \%$ over the 10-day deprivation period during HC$\mathrm{FD}$, the percent ethanol intake rose. Individual correlations between the percent ad-lib body weight and percent ethanol consumed were $r=-.92$ for $A-1$, -.55 for both $\mathrm{A}-2$ and $\mathrm{A}-3$, and -.71 for $\mathrm{A}-4$. The coefficients for A-1 and A-4 were significant $(p<.05$, $\mathrm{df}=8$, one-tailed test). Other studies (Ims, Best, \& Senter, 1976; Palfai \& Reckhow, 1977) have shown similar effects of food deprivation on ethanol consumption.

While food deprivation was manipulated only during home cage choice testing in the present study, there is evidence to suggest that ethanol intake in the SIP paradigm is due to the caloric value of ethanol, rather than its intoxicating effects. For example, Freed and Lester (1970) have demonstrated that polydipsia ethanol consumption continued during extinction of barpressing, whereas intake of water and an equally intoxicating, but noncaloric, acetone solution declined. Also, Freed (1974) has shown that rats (which had polydipsia training with ethanol or water) preferred nonintoxicating, but calorically balanced, butanediol solutions over ethanol. Both of these studies indicate that fooddeprived rats were drinking ethanol primarily for its caloric value during the SIP sessions.

In spite of the return of ethanol drinking during the HC-FD condition in the present experiment, the mean ethanol intake of $34.3 \mathrm{ml}$ does fall $12.3 \mathrm{ml}$ short of that achieved under deprivation conditions in the experimental chamber during polydipsia sessions. Others (Freed \& Lester, 1970; Meisch \& Thompson, 1973) have also noted less ethanol consumption in the experiment chamber with schedule removal. Also, Samson and Falk (1974a) reported greater intake under SIP conditions than in the home cage, under equal levels of food deprivation. Therefore, the role of learned associations between drug ingestion and the stimulus environment in which this behavior is acquired and reinforced (via intoxication or the meeting of nutritional needs) must also be acknowledged as contributing to ethanol intake in the present series of experiments. Previous studies have addressed this possible explanation of ethanol self-administration (Meisch \& Thompson, 1974), and recent reports of increased opiate self-administration under food-deprivation conditions (Carroll, Rance, \& Meisch, 1979; McMillan \& Leander, 1976; Meisch \& Stark, 1977) indicate that interoceptive cues, as well, may enter into association with drug reinforcement.

In conclusion, ethanol intake by laboratory rats in home cage or SIP conditions appears to be largely a function of caloric need, but it may also be influenced by learned associations with the stimulus complex in which drug administration takes place.

\section{REFERENCES}

tion increases oral and intravenous drug intake in rats. Science, 1979, 205, 319-321.

FALK, J. L. Production of polydipsia in normal rats by an intermittent food schedule. Science, 1961, 133, 195-196.

FALK, J. L. \& SAmson, H. H. Schedule-induced physical dependence on ethanol. Pharmacological Reviews, 1976, 27, 449-464.

Falk, J. L., Samson, H. H., \& Winger, G. Behavioral maintenance of high concentrations of blood ethanol and physical dependence in the rat. Science, 1972, 177, 811-813.

Falk, J. L., Samson, H. H., \& Winger, G. Polydipsia-induced alcohol dependency in rats. Science, 1976, 192, 492.

Freed, E. X. Alcohol polydipsia in the rat as a function of caloric need. Quarterly Journal of Studies on Alcohol, 1972, 33, 504-507.

Freed, E. X. Fluid selection by rats during schedule-induced polydipsia. Quarterly Journal of Studies on Alcohol, 1974, 35, 1035-1043.

Freed, E. X., \& Lester, D. Schedule-induced consumption of ethanol: Calories or chemotherapy? Physiology \& Behavior, $1970,5,555-560$.

Heintzelman, M. E., Best, J., \& Singer, R. J. Polydipsiainduced alcohol dependency in rats: A reexamination. Science, $1976,191,482-483$.

ImS, J., Best, J., \& Senter, R. J. Weight reduction and "free choice" polydipsic ethanol consumption. Bulletin of the Psychonomic Society, 1976, 7, 387-389.

KeEhN, J. D., \& Coulson, G. E. Schedule-induced choice of water versus alcohol. Psychological Reports, 1975, 25, 325-328.

Lester, D., \& Freed, E. Z. Criteria for an animal model of alcoholism. Pharmacology, Biochemistry and Behavior, 1973, 1, 103-107.

McMillan, D., \& Leander, J. D. Schedule-induced oral self administration of etonitazene. Pharmacology, Biochemistry and Behavior, 1976, 4, 137-141.

Meisch, R. A., \& Stark, L. J. Establishment of etonitazene as a reinforcer for rats by use of schedule-induced drinking. Pharmacology, Biochemistry and Behavior, 1977, 7, 195-203.

Meisch, R. A., \& Thompson, T. Ethanol as a reinforcer: Effects of fixed-ratio size and food deprivation. Psychopharmacologia, 1973, 28, 171-183.

MEisch, R. A., \& Thompson, T. Ethanol intake as a function of concentration during food deprivation and satiation. Pharmacology, Biochemistry and Behavior, 1974, 2, 589-596.

Mello, N. K. A review of methods to induce alcohol addiction in animals. Pharmacology, Biochemistry and Behavior, 1973, 1,89-101.

Myers, R. K., Stoltman, W. P., \& Martin, G. E. Effects of ethanol dependence induced artificially in the rhesus monkey on the subsequent preference for ethyl alcohol. Physiology \& Behavior, 1972, 9, 43-48.

Palfai, T., \& Reckhow, W. Food deprived-induced alcohol ingestion in the mouse: Calories versus primary sensory preference. Pharmacology, Biochemistry and Behavior, 1977, 6, 709-712.

Rozhrs, T. A., \& Samson, H. H. Schedule-induced ethanol polydipsia: Function of ethanol concentration. Pharmacology, Biochemistry and Behavior, 1980, 13, 291-294.

SAmson, H. H., \& FALK, J. L. Alteration of fluid preference in ethanol-dependent animals. Journal of Pharmacology and Experimental Therapeutics, 1974, 190, 365-376. (a)

Samson, H. H., \& FALK, J. L. Schedule-induced ethanol polydipsia: Enhancement by saccharin. Pharmacology, Biochemistry and Behavior, 1974, 2, 835-838. (b)

TANG, M., \& FALK, J. L. Ethanol dependence as a determinant of fluid preference. Pharmacology, Biochemistry and Behavior, $1977,7,471-474$.

Weste rfeld, W. W., \& LAwrow, J. The effect of caloric restriction and thiamin deficiency on the voluntary consumption of alcohol by rats. Quarterly Journal of Studies on Alcohol, 1953, 14, 378-384. 